\title{
COMPUTING POSITIONS INDICATED BY SPATIAL TERMS IN THREE-DIMENSIONAL SPACE
}

\author{
Takatsugu KOJIMA and Takashi KUSUMI
}

Kyoto University, Japan

\begin{abstract}
Many cognitive studies have examined how to compute, map, or describe the spatial representations of spatial terms. Many of these studies have examined spatial terms that have meanings appropriate for projection onto a two-dimensional upright (2-D) plane. However, the world in which we live and apply spatial terms to is threedimensional (3-D). This study focused on computing the spatial meanings of projective spatial terms on a level plane in 3-D space from an egocentric viewpoint. Three Japanese spatial terms, ushiro, saki, and mae (similar to behind, ahead and far, and in front of, respectively, in English), were examined in three experiments based on 3-D computer graphics virtual space. This study suggested that prototypical points of certain kinds of projective spatial terms such as mae and ushiro can be computed on a level plane in 3-D space using angles defined by viewpoint and reference object positions under the same spatial condition as used in this study.
\end{abstract}

Key words: language and perception, spatial term, spatial cognition, threedimensional space, virtual space

Many studies have examined spatial meanings projected onto a two-dimensional (2D) plane. Few studies, however, have attempted to compute spatial terms onto a level plane either in the real or virtual three-dimensional (3-D) worlds. However, the world in which we live and use spatial terms is a 3-D world. Recent advances in computer graphics (CG) have also made 3-D displays and virtual spaces commonplace. For example, global positioning systems with 3-D CG now assist in-vehicle navigation, and computer-based 3$\mathrm{D}$ maps can help users find a given location within a building or other structure. These systems often use language to provide spatial information. Moreover, certain online chat systems allow users to move and position an "avatar" (a 3-D icon or representation) while interacting with other users in a shared virtual reality space. In these cases, spatial terms must be understood in virtual space. Thus, it is important to examine the relationships between spatial-term apprehension and spatial cognition in a 3-D space, whether it is real or virtual.

\section{Categorizing Space by Language}

We are surrounded by space and categorize it in certain ways, including the use of language. For instance, we may say, "Look at the cat under the table." When you hear

Correspondence concerning this article should be addressed to Takatsugu Kojima, Department of Cognitive Psychology in Education, Graduate School of Education, Kyoto University, Yoshida-honmachi, Sakyo, Kyoto, 606-8501 Japan (e-mail:kjm_fa@nifty.com). 
this sentence, you break the scene into two areas, the area indexed by under and another area. You understand the statement if the cat is in the area that you categorized as under.

Generally, the spatial relationships predicated by spatial terms involve two objects: a located object and a reference object (Landau \& Jackendoff, 1993; Miller \& JohnsonLaird, 1976). For example, in the statement "A is above B," A is the located object and B is the reference object.

Many studies have examined how to compute, map, or describe the relationships between spatial terms and spatial representations (Carlson-Radvansky \& Logan, 1997; Hayward \& Tarr, 1994; Herskovits, 1986; Kreizer, 1997; Logan \& Sadler, 1996; Regier, 1996; Regier \& Carlson, 2001; Talmy, 1983). Research has found that the reference frame and spatial template are important factors in computing the apprehension of spatial relationships.

\section{Reference Frame}

The reference frame is a 3-D coordinate system with four parameters: origin, orientation, direction, and scale (Logan \& Sadler, 1996). The origin is the intersection of three axes and located at the center of the reference object. The orientation defines the orientations of the three axes (e.g., above/below, left/right and in front of/behind). The direction defines the direction of each axis, e.g., which direction is above in the above/ below axis. The scale defines the units of distance on the axes. The reference frame underlies the alignment of the spatial template.

\section{Spatial Template}

Spatial templates visually represent the spatial categories of a spatial term relative to a reference object. Spatial templates consist of good, acceptable, and bad regions in terms of the spatial relation predicated by a spatial term (Logan \& Compton, 1996; Logan \& Sadler, 1996).

Fig. 1 shows typical spatial templates based on those by Hayward and Tarr (1995) for above and below relative to $R$, which is the reference object in cell $(4,4)$. The darker area shows the area of higher ratings for the spatial terms. As Fig. 1 shows, the acceptability ranges of above and below are highest in regions along the vertical line from the reference object. These regions are referred to as the good regions. In general, the good regions of axial or projective spatial terms (e.g., above and below) are linear. Similar results have been reported in other studies (Carlson-Radvansky \& Logan, 1997; Crawford, Regier, \& Huttenlocher, 2000; Kojima, 2003; Logan \& Sadler, 1996; Munnich, Landau, \& Dosher, 2001). If necessary, the most acceptable point in a good region of a spatial term can be set. Two other important terms (prototypical and ideal points) are also associated with the mapping of a spatial term; here, we define these terms based on the work of Herskovits (1986). A prototypical point is the most acceptable point of a spatial template for a specific situation. An ideal point is a more abstract point derived from prototypical points. These two points are important to understand or compute spatial terms.

According to Logan and Sadler (1996), in apprehending a spatial relationship predicated by a spatial term, a reference frame must be superimposed on or extracted from 


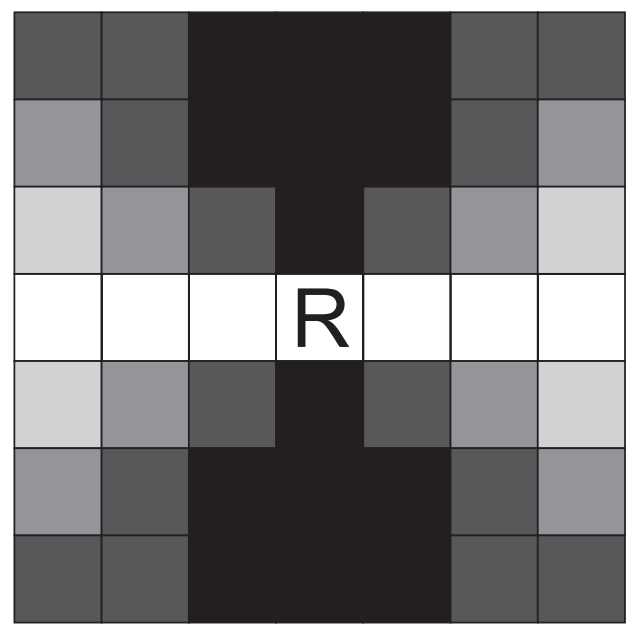

Fig. 1. Typical examples of acceptability distributions for above (upper) and below (lower). $R$ indicates the reference object.

a reference object. A spatial template must then be superimposed on the reference object and aligned with the reference frame in a representational space based on an observed or imagined scene. Then, it is possible that goodness-of-fit computation may be applied to the spatial template. In the goodness-of-fit computation, it is assumed that the prototypical point is determined and then the overall distribution pattern of the acceptability spreads based on the prototypical point.

Spatial templates and computing goodness of fit on them have several particular characteristics. The overall shapes of spatial templates are not necessarily specific to the located objects (Carlson-Radvansky \& Logan, 1997; Hayward \& Tarr, 1995; Logan \& Sadler, 1996). The goodness-of-fit computation is thought to be independent of referenceframe processes (Carlson-Radvansky \& Logan, 1997; Logan \& Sadler, 1996). Further, the function of the reference object influences the acceptability range of the distribution pattern of spatial templates (Carlson-Radvansky, Covey, \& Lattanzi, 1999). An extra object that is neither a located nor a reference object reduces the acceptability on a spatial template (Carlson \& Logan, 2001). Good regions of projective spatial terms cannot always be described by linear areas or a line when an extra object is present (Kojima, 2003; Kojima \& Kusumi, 2002).

Although these findings are important, most previous studies on spatial templates or spatial categorization using language have used 2-D visual stimuli (cf. Franklin, Henkel, \& Zengas, 1995; Imai, Nakanishi, Miyashita, Kidachi, \& Ishizaki , 1999). Similarly, research on spatial-term apprehension has used 2-D upright planes, such as 2-D displays on CRT screens that lack cues for 3-D spatial perceptions.

\section{Computing a Prototypical Point on a Spatial Template on a Level Plane in 3-D Space}

In 3-D space, as perspectives change with viewing distance, height, and angle, 


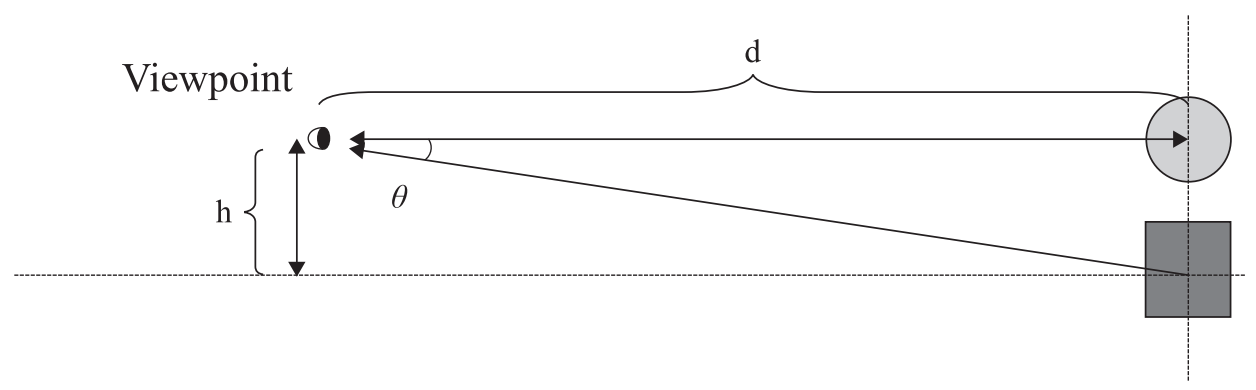

Fig. 2. Parameters for computing the ideal position of a located object indexed by a spatial term. The sphere is the reference object, and the cube is the located object. We defined the positions of the reference and located objects by their central points. The viewpoint height was assumed to be the height of the reference object center. $d$ represents the distance between the viewpoint and the 2-D plane containing the reference and located objects, $h$ is the viewpoint height based on a line through the reference object center, and $\theta$ is the angle formed between the line through the viewpoint and the reference object center and the line through the viewpoint and the located object center.

observed scenes also change. Viewpoint change will have a greater influence on an observed scene when objects are arrayed on a level plane in 3-D space (e.g., ground plane) than on an upright plane in 2-D or 3-D.

In studying the visual perception of length, Norman, Todd, Perotti and Tittle (1996) showed that viewers could correctly perceive a 2-D length on an upright plane using Euclidean techniques. However, length perception in 3-D space was influenced by depth cues; viewers could not as correctly estimate a 3-D length in a Euclidean manner, either in 3-D CG space on a computer screen or in the real world. According to previous research on visual perception of length in 3-D space, distances in empty space (Norman \& Todd, 1998; Norman et al., 1996) or on a ground plane in a 3-D space (Loomis, Da Silva, Fujita, \& Fukushima, 1992; Wagner, 1985) are perceived as shorter than the actual physical distances. Moreover, the ability to discriminate between length differences in 3-D space varies and is systematically influenced by viewing distance (Norman et al., 1996). This viewing-distance effect on perceived distances in 3-D space has been observed for both flat and curved surfaces (Norman, Norman, Lee, \& Stockton, 2004; Norman, Lappin, \& Norman, 2000).

Thus, various factors can distort the perceived distance between a reference and located objects in a 3-D scene. As noted above, viewers can correctly detect a 2-D length using Euclidean methods. The prototypical point of a spatial term can therefore be computed by its absolute distance (the visual angle) from a reference object based on a perceived scene, as expressed in the equation below:

$$
h=d \tan \theta
$$

Here, $h, d$, and $\theta$ are the parameters shown in Fig. 2.

However, perception of 3-D length can be distorted and rendered incomputable by Euclidean methods. How, then, can we determine a prototypical point based on a distorted distance? 
Data from a spatial template of a spatial term on a 2-D upright plane can be applied to other 2-D upright planes by linear mapping. Previous studies have proven the appropriateness of this technique both intuitively and psychologically. However, can we use the same method to apply a spatial template based on a level plane to other level planes in 3-D space?

Previous research on egocentric distance perception in 3-D space (Ooi, $\mathrm{Wu}, \& \mathrm{He}$, 2001; Philbeck \& Loomis, 1997) has suggested that the angle between an observer's eye level and an object position provides strong cues for the horizontal distance between observer and object. According to Creem-Regehr, Willemsen, Gooch, and Thompson (2005), an observer's ability to see his/her body and feet does not affect accurate distance perception. We therefore hypothesized that an ideal position of a projective spatial term could be computed using the angle between an observer's eye position (viewpoint) and the positions of reference and/or located objects if the ideal position was on a projective line on a plane passing through positions of the observer and the reference object. We refer to this kind of projective spatial term as a depth-dependent projective spatial term.

In this study, we focused on how to map computationally ideal points of depthdependent projective spatial terms in 3-D space; we then assumed that relative relationships between viewpoint and object-position angles would be important in computing the prototypical position of a projective depth-dependent spatial term on a level plane in 3-D space. We examined which factors were important in computing these ideal points. We used 3-D CG space ${ }^{1}$ and three Japanese depth-dependent projective spatial terms appropriate for the goal of this study, ushiro, saki and mae (basically similar to behind, ahead and far, and in front of, respectively in English $)^{2}$.

In Experiment 1, the spatial templates of ushiro and saki under the spatial condition of this study were examined to clarify their slight and important difference. In Experiment 2, we examined the relationship between their prototypical points on a level plane in 3-D space using the method of adjustment, and hypothesized a computational model on a prototypical point of a certain kind of depth-dependent projective spatial term. Experiment 3 replicated Experiment 2 using mae and confirmed the assumption obtained from Experiment 2.

\section{EXPERIMENT 1}

In Experiment 1 and 2, we chose two Japanese spatial terms, ushiro and saki, which are typical depth-dependent projective spatial terms in Japanese. They are commonly used to index objects located on a level plane in 3-D space and share almost the same spatial

\footnotetext{
${ }^{1}$ The 3-D CG world is obviously not the real world; however, this does not affect our results because distance is generally underestimated in a 3-D CG world, and this underestimation is systematic and stable for each individual viewer (Armbrüster, Herber, Valvoda, Kuhlen, \& Spijkers, 2005).

${ }^{2}$ Ushiro, mae and saki are polysemic words and have various meanings, apart from their spatial meanings. In addition, their spatial meanings also have variety. However, we limited their use to depth-dependent projective spatial meanings under a certain reference frame and egocentric viewing conditions in this study.
} 


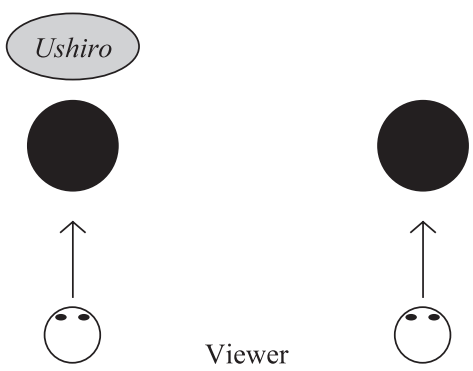

Fig. 3. Schematic images of ushiro and saki. The black circle is the reference object. The gray areas show areas indexed by ushiro and saki.

template pattern and good region under a spatial condition like this study. However, they are assumed to differ from each other in their ideal points on the good region.

Ushiro indexes an area nearer to a reference object and becomes less acceptable if a located object is set farther from the reference object. Saki indexes an area farther from a reference object and becomes more acceptable even if a located object is set relatively far away from the reference object. Fig. 3 shows typical schematic images of areas indexed by ushiro and saki and an important difference between their spatial meanings under the same spatial condition as used in this study. Basically, under such conditions, the ushiro area is limited by the reference object position, while the saki area is not. Moreover, although saki can be used even if the located object cannot be observed in a scene, it must be located within an area on an axis extending from the reference object. In English, ushiro and saki may be loosely translated as behind or in back of and ahead and far, respectively, in this study.

In a computational research on an ideal point of a spatial term in Japanese, Tokunaga, Koyama and Saito (2005) divided Japanese spatial nouns into two major classes: part and apart. The part class designates a part of a reference object. The apart class designates a location apart from a reference object. Moreover, they divided the apart class into two subclasses: distance-type and direction-type. According to their classification, ushiro and mae have the characteristics of the part class and direction-type apart class, and saki has the characteristics of the part class and distance-type apart class ${ }^{3}$.

\footnotetext{
${ }^{3}$ There will be other criteria for classification of spatial meanings of ushiro, mae and saki. For example, from the viewpoint of the reference frame, it can be presumed that ushiro and mae are related to the objectcentered reference frame, and that saki is related to the egocentric reference frame. Focusing on the direction of the reference object, the inherent front of a reference object will have more effect on ushiro and mae than on saki. We do not preclude these ways of classification, but we referred to the geometric classification of Tokunaga et al. (2005) for the goal of this study.
} 


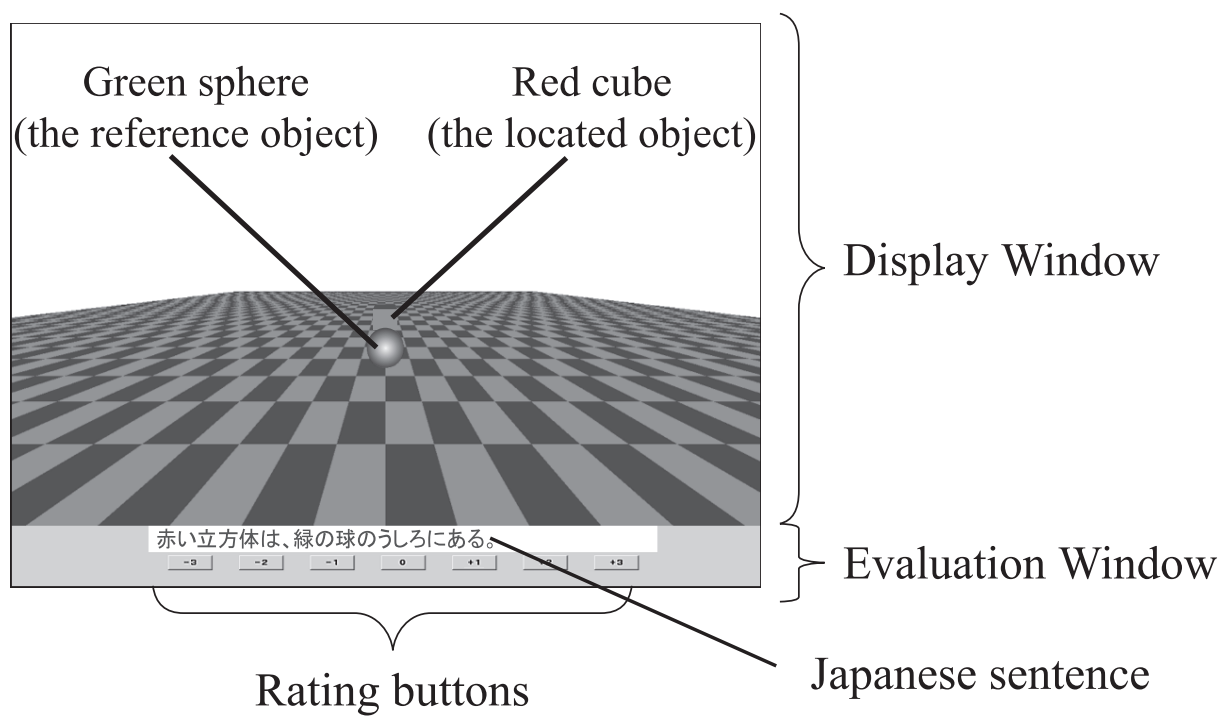

Fig. 4. Example of a display in Experiment 1. The upper window is the display widow and the lower window is the evaluation window.

Do the intuitive or typological difference of characteristics of ideal points between ushiro or mae and saki influence our assumption on computing the prototypical position of a projective depth-dependent spatial term on a level plane in 3-D space? To examine this point, we used ushiro and saki in this study at first. However, the spatial templates of these terms may be difficult for non-native Japanese speakers to visualize or apprehend. Furthermore, although most native Japanese are assumed to understand that ushiro and saki share nearly the same good region, given the spatial situations of this study, no empirical data yet support this assumption. Therefore, Experiment 1 was conducted to make the spatial template more accessible to non-native Japanese speakers and to provide empirical support for the assumed spatial template.

\section{METHOD}

Participants. Thirty graduate and undergraduate students at Kyoto University participated in Experiment 1. All were native Japanese speakers.

Materials. The experiment was conducted on a computer with a 17 -inch monitor. Two windows appeared on the monitor screen (Fig. 4). The display window appeared at the top of the screen and presented the stimuli. The evaluation window appeared below the display window; this window presented statements and received responses. The stimuli were constructed using Open GL ${ }^{\circledR}$ (Silicon Graphics, Inc., Mountain View, CA, USA), a high-performance platform for 3-D graphics. The stimuli were presented in a 3-D CG space from an egocentric view. The stimuli consisted of a floor, a green sphere, and a red cube. The evaluation window included an area for presenting statements (in Japanese for all experiments), a button to change the stimuli in the display window and the panel status in the evaluation window, and seven rating buttons representing a scale from -3 (least applicable) to +3 (most applicable). A value of zero indicated fair or moderate. The sentences used in this experiment were "Akai rippoutai-wa midori-no kyuu-no ushiro-ni aru" ("The red cube is behind the green sphere") or "Akai rippoutai-wa midori-no kyuu-no saki-ni aru" ("The red cube is ahead and far from the green sphere"). 


\section{Viewpoint}

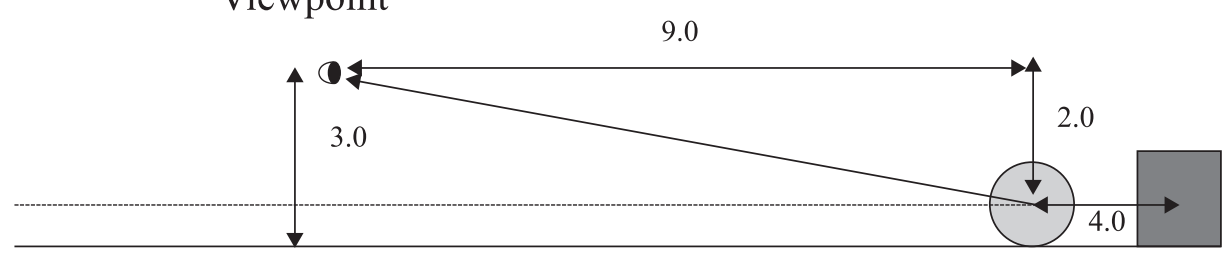

Fig. 5. View positions and object locations in 3-D CG space. Each value is based on a coordinate value in 3-D virtual space.

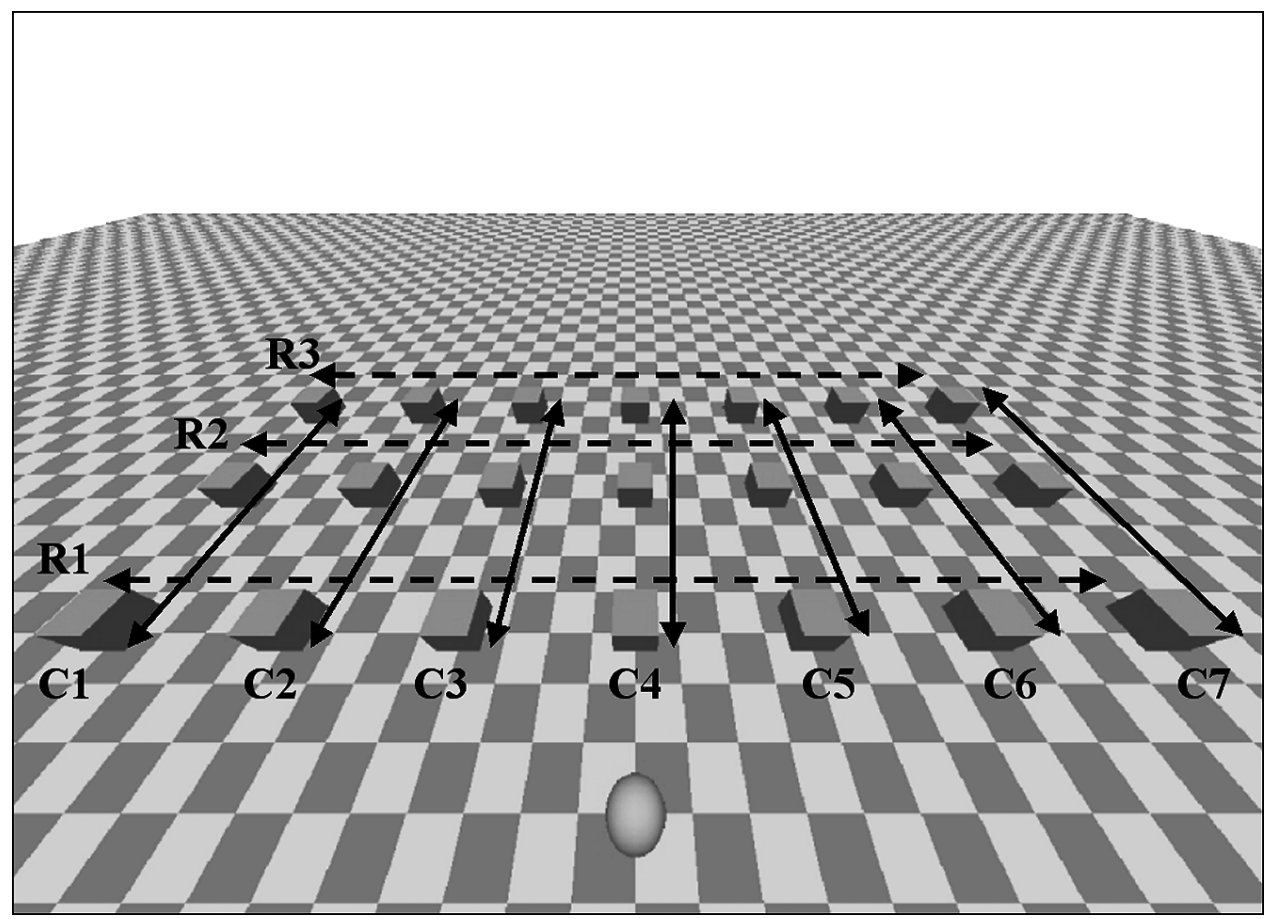

Fig. 6. Locations used in Experiment 1. Each cube represents one object location. The view angle and lighting of this figure differ from those used in the experiment to simplify and clarify location representations. R1-R3 with dotted arrows and $\mathrm{C} 1-\mathrm{C} 7$ with solid arrows represent longitudinal and lateral locations respectively.

The positions of the reference object (the green sphere) and the viewpoint were fixed at their respective coordinates in the 3-D CG space (Fig. 5). The position of the located object (the red cube) was fixed at one of 21 locations in the 3-D CG space (Fig. 6) and the frame of reference was also fixed. The floor consisted of light or dark gray panels and the panel combination pattern was randomly constructed in each trial so that participants would not use the panel patterns as visual cues. The reference frame was fixed to a specific and appropriate frame during the experiment.

We used 42 different stimuli (two statements $\times 21$ target locations) in this experiment.

Procedure. Each participant viewed the computer screen from a distance of approximately $115 \mathrm{~cm}$ in a dark room. In each trial, a blank white space appeared in the display window and a statement was presented 


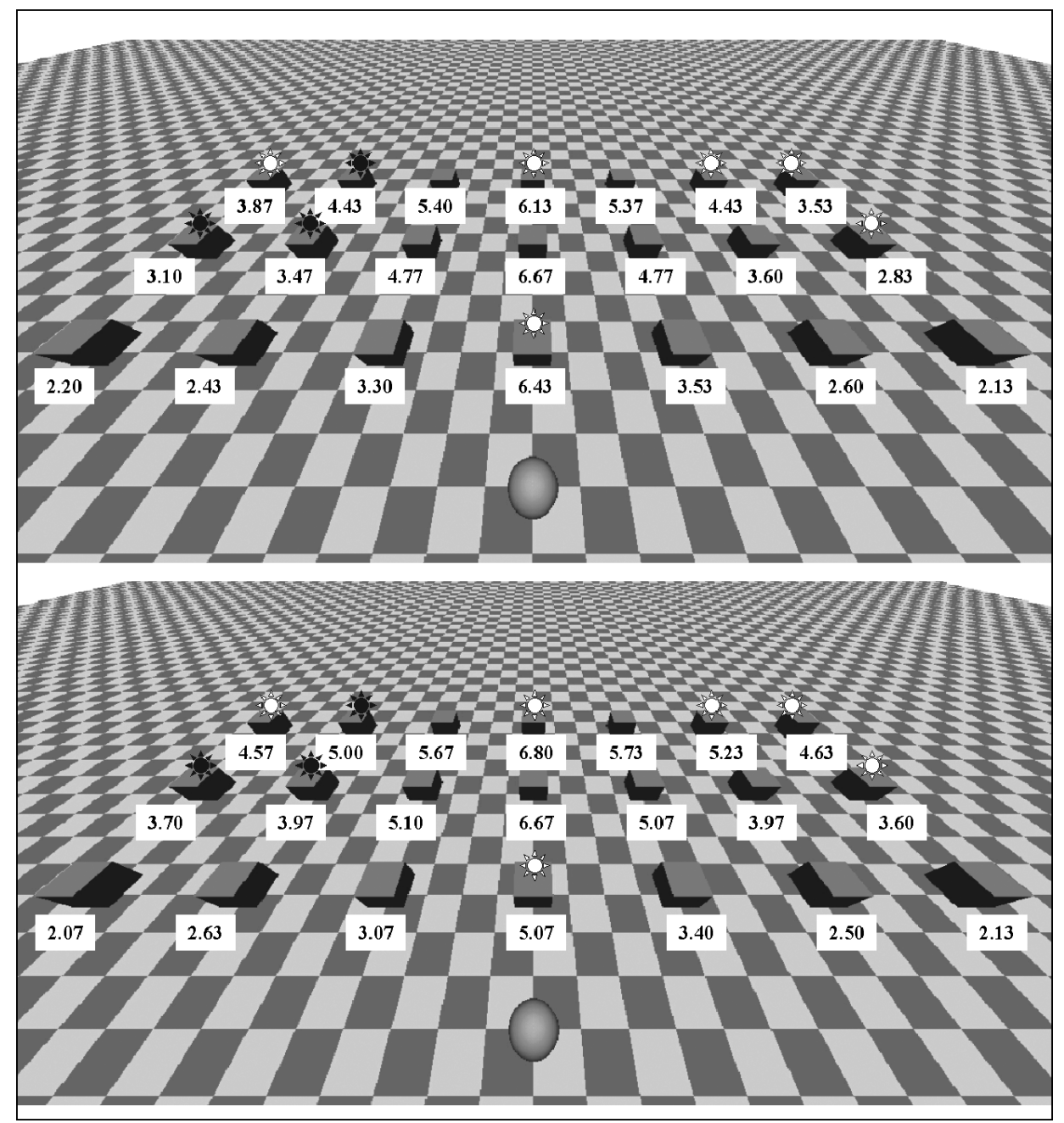

Fig. 7. Mean acceptability rating of ushiro (top) and saki (bottom) for each location in Experiment 1 . The black and white characters on the cubes indicate statistically significant differences $(p s<.05$ and .01 respectively) between ushiro and saki.

in the evaluation window. The participants read the sentence and then clicked on the button in the evaluation window using the computer mouse to present the stimulus in the display window. The experiment was explained to all of the participants, who completed five practice trials before participating in the 42 experimental trials. The presentation order was generated randomly for each participant. This experiment was similar to the experiment described by Hayward and Tarr (1995).

\section{RESULTS}

Fig. 7 shows the mean acceptability rating for each location under a given condition. The ratings from -3 to +3 were transformed into values from 1 to 7 .

The mean acceptability values for each location, for ushiro and saki were subjected 
to a three-way 2 (spatial term: ushiro and saki) $\times 3$ (longitudinal location of the located object) $\times 7$ (lateral location of the located object) repeated measures analysis of variance (ANOVA). There were significant main effects of spatial terms $(F(1,29)=7.31, p=.011$, $M S E=3.03)$, the longitudinal location of the located object $(F(2,58)=102.37, p<.0001$, $M S E=4.02)$, and the lateral location of the located object $(F(6,174)=107.47, p<.0001$, $M S E=2.02)$. There were also two-way interactions between the spatial term and longitudinal location of the located object $(F(2,58)=12.77, p<.0001, M S E=1.76)$, the spatial term and lateral location of the located object $(F(6,174)=4.57, p=.0002$, $M S E=0.74)$, the longitudinal and lateral locations of the located object $(F(12$, $348)=5.50, p<.0001, M S E=1.03)$, and the three-way interactions $(F(12,348)=1.87$, $p=.0370, M S E=1.87$ ).

To show more clearly differences between ushiro and saki, we conducted analyses as follow.

First, we analyzed simple main effects of the spatial term in interactions between the spatial term and longitudinal location, and the spatial term and lateral location. Here, we set the three longitudinal locations as three rows from R1 (upper) to R3 (lower), and the seven lateral locations as seven columns from C1 (left) to C7 (right) (see Fig. 6). There were significant simple main effects of the spatial term at $\mathrm{R} 2(F(1,87)=8.07, p=.0056$, $M S E=2.18)$ and R3 $(F(1,87)=19.59, p<.0001, M S E=2.18)$ in the spatial term $\times$ longitudinal location interaction and at $\mathrm{C} 1 \quad(F(1,203)=6.36, p=.0124$, $M S E=1.07), C 2 \quad(F(1,203)=8.02, p=.0067, M S E=1.07), \mathrm{C} 6 \quad(F(1,203)=5.32$, $p=.0221, M S E=1.07)$, and C7 $(F(1,203)=16.30, p=.0001, M S E=1.07)$ in the spatial term $\times$ lateral location interaction.

Then, simple interaction effects between the longitudinal and lateral locations were analyzed for the spatial term. There was a significant simple interaction effect only for ushiro $(F(12,696)=6.37, p<.0001, M S E=0.86)$.

Finally, simple simple main effects of the spatial term at each combination of longitudinal and lateral locations were analyzed (see Fig. 7). Here, we set the upper-left location as cell $(1,1)$ and the lower-right location as cell $(3,7)$ because the locations of the located objects were considered as a three-by-seven grid. There were significant simple simple main effects of the spatial term at nine locations; cell $(1,1)(F(1,609)=8.03$, $p=.0048)$, cell $(1,2)(F(1,609)=5.26, p=.0222)$, cell $(1,4)(F(1,609)=7.28$, $p=.0072)$, cell $(1,6)(F(1,609)=10.48, p=.0013)$, cell $(1,7)(F(1,609)=19.82$, $p<.0001)$, cell $(2,1)(F(1,609)=5.90, p=.0155)$, cell $(2,2)(F(1,609)=4.10$, $p=.0435)$, cell $(2,7)(F(1,609)=9.63, p=.0020)$, and cell $(3,4)(F(1,609)=30.60$, $p<.0001)$. All the MSEs were 0.92 .

\section{DISCUSSION}

The results indicated that ushiro and saki are depth-dependent projective spatial terms that shared almost the same good region under the experimental conditions. The analyses of simple simple main effects showed that the acceptable region of ushiro was slightly narrower than that of saki on the whole. From the mean acceptability data and the analyses of the simple main effects and simple interaction effect, the ideal location of 
ushiro was assumed to be closer to the reference object and closed around it, and the ideal location for saki was assumed to be farther from the reference object. These assumptions correspond to our assumption of the basic difference between ushiro and saki, i.e., the ushiro area would be limited by the position of the reference object, but the saki area would not be, and would signify an area relatively farther away from the reference object. The results of Experiment 1 correspond to the schematic images in Fig. 3. Here, we define two types of depth-dependent projective spatial terms for convenience in later discussions: open-area and closed-area projective spatial terms. An open-area projective spatial term indexes an area on an axis expanding from a reference object and the area or position of the located object does not have to be observed in the visual field. A closed-area projective spatial term indexes an area on an axis expanding from a reference object and the area or the position of the located object is limited by the reference object. Therefore, ushiro is a closed-area spatial term and saki is an open-area spatial term. This classification is definitive but consistent with a more general or abstract classification of Japanese spatial nouns (Tokunaga et al., 2005).

Although Experiment 1 provided an overview of the spatial templates of ushiro and $s a k i$, the experiment did not deliver enough detailed information about the relationship between the prototypical points of ushiro and saki. Therefore, we conducted Experiment 2 to examine the relationship between the prototypical points of ushiro and saki and the factors important for computing ideal points.

\section{EXPERIMENT 2}

Because a 2-D length can be correctly detected in a Euclidean manner (Norman et al., 1996), a prototypical point or location of a projective spatial term can be computed by the absolute distance (the visual angle) from a reference object. However, a 3-D length is distorted, and cannot be computed using Euclidean references (Norman et al., 1996; Norman et al., 2000; Norman et al., 2004). In a 3-D space, the scene appearance changes with viewpoint changes. Thus, it is inappropriate to turn a 3-D view into a 2-D map based on the 2-D visual appearance or to compute distance based on objects on the 2-D map. However, this concern does not apply to some projective spatial terms such as left and right, which can be depicted in their ideal positions on a 2-D upright plane and for which horizontal length can be detected comparatively correctly even in 3-D space (Norman et al., 1996). However, if we compute prototypical positions of projective spatial terms depicted on a level plane in 3-D space, we must apply factors other than distance to compute prototypical positions of projective spatial terms.

According to Norman et al. (1996), viewing distance can greatly affect the perception of depth-dependent length in a 3-D space; in that study, participants' judgment of 3-D length were inaccurate, as a whole, but each subject had subjective patterns of judgment. That is, the visual perception of 3-D length was incorrect but stable individually or relatively (see also Armbrüster et al., 2005). Previous studies have also suggested that the angle between an observer's eye level and an object position at the 
ground plane in 3-D space can be strong cues for accurately computing the horizontal distance between observer and object (Ooi et al., 2001; Philbeck, \& Loomis, 1997). Furthermore, accurate distance perception does not require that viewers see their own bodies or feet (Creem-Regehr et al., 2005).

From the above studies, we assumed that relative relationships between viewpoint and object-position angles would be important in computing the prototypical position of a projective depth-dependent spatial term on a level plane in 3-D space. In this experiment, we focused on the relationship between viewpoint, positions of reference and located objects, and the prototypical points of ushiro and saki. We used the method of adjustment to examine the relationship in detail.

The viewing distance and the following three factors served as the experimental adjustment parameters: the distance between the reference and located objects (located object adjustment condition), the distance between the viewpoint and the reference object (distance adjustment condition) and the height of the viewpoint (height adjustment condition).

If each prototypical point of each spatial term has a specific relationship to viewpoint or the reference or located object positions, we can compute prototypical points of depthdependent projective spatial terms. Moreover, we should be able to identify fixed relationships between the ideal positions of ushiro and saki.

\section{METHOD}

Participants. Thirty Japanese graduate or undergraduate students at Kyoto University participated in Experiment 2. All were native Japanese speakers who had not participated in Experiment 1. The students were divided into two groups of 15 people each, with each group assigned a spatial term (ushiro or saki).

Materials. The experiment was run on a computer with a 17-inch monitor. Two windows were shown on the screen: the display window appeared at the top of the screen and was used to present the stimuli; the adjustment window appeared below the display window (Fig. 8). The stimuli on the display window were constructed as in Experiment 1.

In each trial, the adjustment window showed one of three scroll bars used to adjust three corresponding conditions: located object adjustment condition, distance adjustment condition and height adjustment condition. The main conditions of interest were the located object adjustment and viewing distance adjustment conditions, while the height adjustment condition was used as a "filler condition" to make it difficult for participants to recognize the experimental purpose. In the located object adjustment condition and the height adjustment condition, three viewing distance conditions (near, middle and far) were prepared (Fig. 9). The middle position was at the same position as in Experiment 1. Thus, we used four experimental patterns and three filler patterns. A statement was then presented in the window during an experimental session to remind participants of their requirements. We used the same floor, sentences, and reference and located objects as in Experiment 1. When a participant adjusted one factor, the coordinates for the other two factors remained fixed in the 3-D CG space.

For each experimental trial, the default value for the adjustment-window scroll bar was set to the highest or lowest value. A total of 70 experimental trials (seven patterns $\times$ two default values $\times$ five repetitions) were conducted.

Procedure. Each participant viewed the computer screen from a distance of approximately $115 \mathrm{~cm}$ in a dark room. In each trial, one of the three scroll bars and a statement were presented in the adjustment window. All participants were required to slide the bar, using the mouse to adjust the display-window scene so that it matched the statement. All participants were given instructions and were allowed seven practice trials to ensure that they understood the procedures. Following the practice trials, each participant completed 70 experimental trials. Presentation order was generated randomly for each participant. 


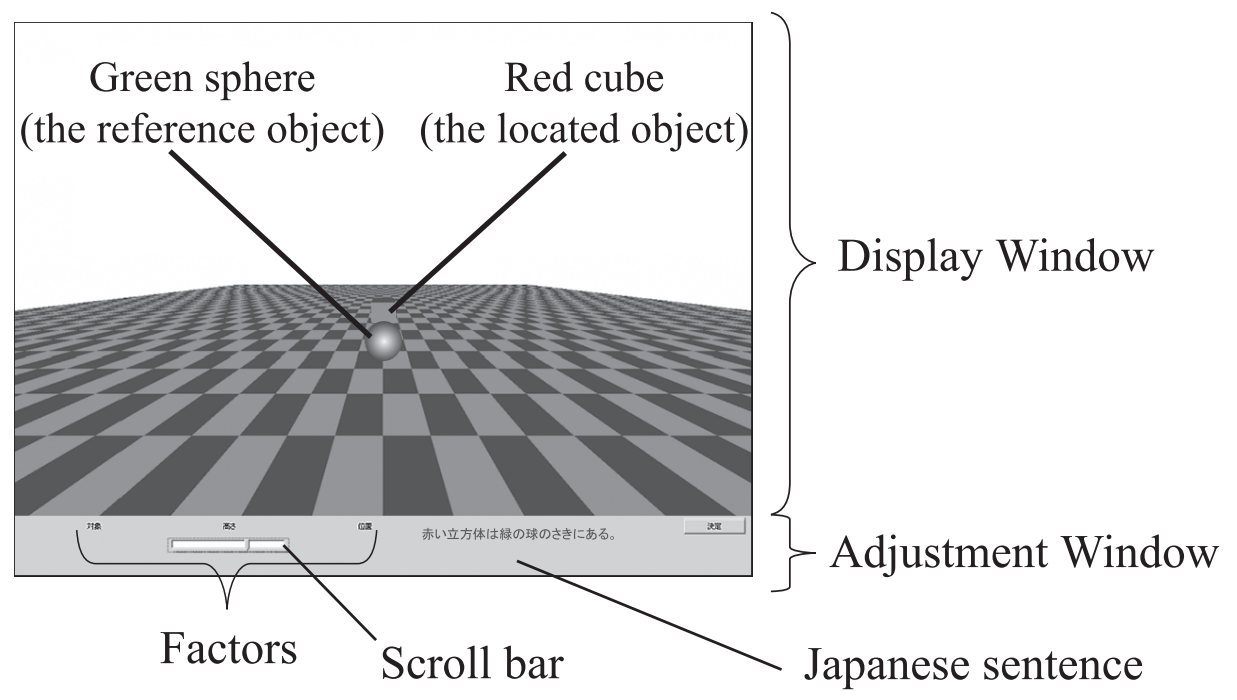

Fig. 8. Example of a display in Experiment 2. The upper window is the display window and the lower window is the adjustment window.

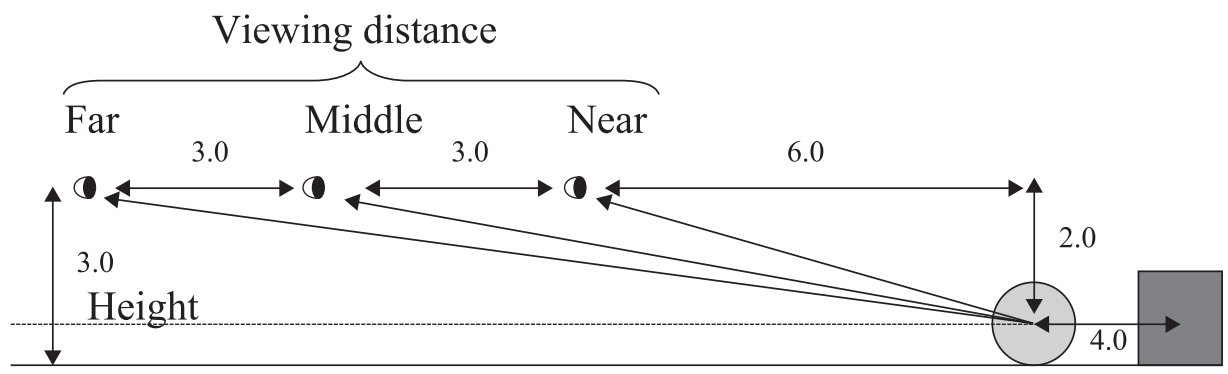

Fig. 9. Controlled factors in Experiment 2. Each value is based on a coordinate value in 3-D virtual space.

\section{RESULTS}

All the raw data were saved as coordinate values in 3-D CG space. We used the data to calculate viewing distances from the reference object in the distance adjustment condition and distances between reference and located objects in the located object adjustment condition.

First, we analyzed the difference between the prototypical positions of ushiro and saki. For the located object adjustment condition, a two-way 2 (spatial term: ushiro and saki) $\times 3$ (viewing distance: near, middle, and far) mixed ANOVA on the distance between reference and located objects was performed with the spatial term as a betweensubject variable and the viewing distance as a within-subject variable. Significant main effects were found for both spatial terms, $F(1,28)=45.70, p<.0001, M S E=14.303$, and the viewing distance, $F(2,56)=15.57, p<.0001, M S E=2.733$; however, no significant 


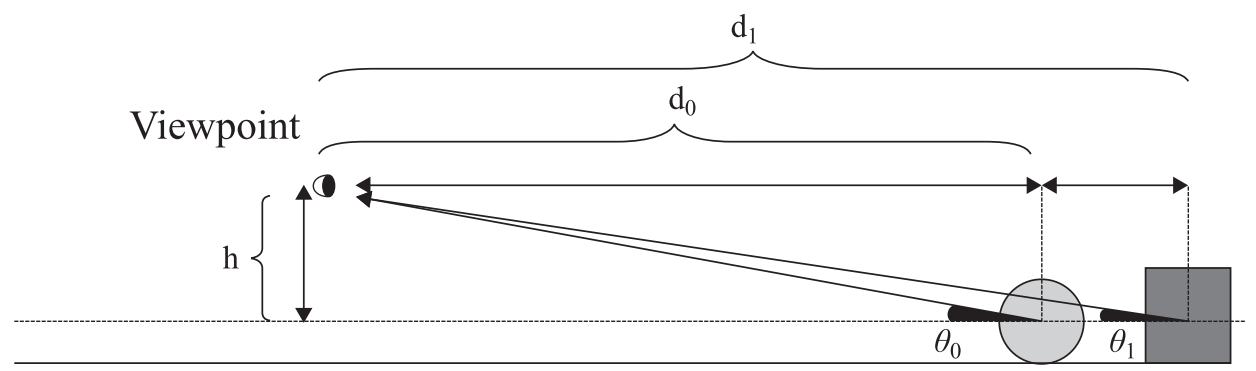

Fig. 10. Parameters for computing the ideal position of a located object indexed by a spatial term on a level plane in 3-D space. The sphere is the reference object, and the cube is the located object. We defined the positions of the reference and located objects by their centers. The viewpoint height is the height from the object center. $d_{0}$ or $d_{1}$ are the horizontal distances between the viewpoint and the reference object $\left(d_{0}\right)$ or located object $\left(d_{1}\right), h$ is the viewpoint height from a line through the reference object center, and $\theta_{0}$ and $\theta_{1}$ are the angles formed between the line through the viewpoint and reference object center (for $\theta_{0}$ ) or the located object center (for $\theta_{1}$ ) and the line through the viewpoint and located object center.

interaction was found. As a post-hoc test, we conducted a Bonferroni test at a significance level of .01 for the viewing-distance factor. Significant differences in all combinations of view positions were revealed for both spatial terms.

For the distance adjustment condition, a $t$-test on the viewing distance from the reference object revealed a significant difference between ushiro and saki $(t(28)=9.00$, $p<.0001)$.

We then converted all the raw data into angle data by calculating ascending vertical angles from the center of the located object to the viewpoint positions and from the center of the reference object to the viewpoint positions (see Fig. 10). The mean angle values in each condition were computed from the angle data. We defined the mean angle values as the mean points of subjective equalities (PSEs) on angle (Table 1, 2, and 3).

We computed ratios between the angles of reference and located objects for ushiro and saki. These ratios were subjected to two one-way repeated measures ANOVAs on the viewing distance by bundling the located object adjustment and distance adjustment conditions together; the viewing distance adjustment condition data were then treated as a fourth data type (in addition to near, middle, and far) in the located object adjustment condition ${ }^{4}$. For ushiro, we found no significant main effect of viewing distance. Thus, the ratio for ushiro was stable, $F(3,42)=1.81, p=.1602, M S E=0.003$. For $s a k i$, we found a significant main effect of viewing distance, $F(3,42)=38.68, p<.0001, M S E=0.002$, and then conducted a Bonferroni test at a significance level of .01 as a post hoc test. We found significant differences in all combinations of viewing distances except for a combination of near and middle in the located object adjustment condition.

\section{DISCUSSION}

The two-way mixed measure ANOVA indicated that the prototypical point of ushiro differed from that of saki, and that participants could distinguish ushiro from saki properly 
Table 1. Mean (SD) PSEs in the located object adjustment condition (degrees)

\begin{tabular}{cccc}
\hline \multirow{2}{*}{ Spatial term } & \multicolumn{3}{c}{ View position } \\
\cline { 2 - 4 } & Near & Middle & Far \\
\hline ushiro & $12.13(1.93)$ & $8.37(1.34)$ & $6.61(0.92)$ \\
saki & $8.22(1.20)$ & $6.19(0.86)$ & $4.98(0.58)$ \\
\hline
\end{tabular}

Note. These values represent means of the angles shown as $\theta_{1}$ in Fig. 10.

Table 2. Mean $(S D)$ ratios for the located object adjustment condition

\begin{tabular}{cccc}
\hline \multirow{2}{*}{ Spatial term } & \multicolumn{3}{c}{ View position } \\
\cline { 2 - 4 } & Near & Middle & Far \\
\hline ushiro & $0.68(0.11)$ & $0.67(0.11)$ & $0.70(0.10)$ \\
saki & $0.43(0.08)$ & $0.49(0.07)$ & $0.52(0.06)$ \\
\hline
\end{tabular}

Note. These values represent mean ratios of $\theta_{1}$ to $\theta_{0}\left(\theta_{1} / \theta_{0}\right)$, the angles shown in Fig. 10 .

Table 3. Mean $(S D)$ PSEs and ratios for the distance adjustment condition

\begin{tabular}{crcc}
\hline \multirow{3}{*}{ Spatial term } & \multicolumn{3}{c}{ PSE (degrees) } \\
\cline { 2 - 4 } & to Located object & to Reference object & Ratio \\
\hline ushiro & $8.23(1.11)$ & $11.61(2.23)$ & $0.72(0.04)$ \\
saki & $11.85(1.10)$ & $20.03(2.95)$ & $0.60(0.04)$ \\
\hline
\end{tabular}

Note. These values represent mean PSEs of $\theta_{1}$ (to the located object) and $\theta_{0}$ (to the reference object) for angles $\theta_{0}$ and $\theta_{1}$ shown in Fig. 10. Ratio values indicate mean ratios of $\theta_{1}$ to $\theta_{0}\left(\theta_{1} / \theta_{0}\right)$.

in the experiment.

The one-way repeated measure ANOVA on the ratios between angles from the viewpoint positions to the reference and located object positions indicated that the prototypical position of ushiro was relatively fixed to the reference object but that the prototypical position of saki was not fixed. This difference between ushiro and saki reflects the fact that the position of the reference object defined the ideal point for ushiro but did not necessarily define the ideal point for saki. Experiment 1 results (Fig. 7) show that a narrower area was indexed for ushiro than for saki, and that the prototypical position of ushiro was closer to the reference object than that of saki.

The common spatial meaning of saki as a depth-dependent projective spatial term under a spatial condition like the experiments was assumed to index a farther point or area 
around a vanishing point. That is, saki seemed to be independent of the reference object position but dependent on a vanishing point. In contrast, ushiro is not used if the point indexed by ushiro is relatively far from a reference object. Table 3 shows that for saki, participants would adjust the viewpoint position closer to the reference object than for ushiro. Positions of the reference and located objects were fixed in the distance adjustment condition. Thus, if a viewpoint position was closer to the reference object, the apparent distance between the reference and located objects would appear longer; the apparent distance between the reference object and a vanishing point would also appear longer than the apparent distance between the located object and the vanishing point. These results suggest that the ideal point of saki could depend not only on a vanishing point but also on the distance from a reference object and viewpoint ${ }^{5}$.

The prototypical point of ushiro was supposed to be defined by the viewpoint and reference object positions. As Table 2 and 3 show, the mean ratios for each viewing distance condition for ushiro were $0.68,0.67,0.70$ and 0.72 respectively. The mean ratio of the four ratios was 0.69 . In a 3-D CG space, it is possible to determine coordinate values for a viewpoint position and the center of a reference object. Therefore, we can compute the prototypical position of ushiro to the reference object from the equation below, at least for conditions similar to those in this experiment:

$$
d_{n}=h \cot \left(\theta_{0} / 0.69\right)
$$

In the above equation, $h$ and $\theta_{0}$ are the parameters shown in Fig. 10 and $d_{n}$ is a predicted position of a prototypical point.

In this experiment, the objects were arrayed on a plane in 3-D space and observed scenes changed as perspective changed with viewing distance. Nevertheless, the specific relationship of the prototypical point of ushiro, the viewpoint and reference object positions indicated that it was possible to compute a prototypical point for a closed-area projective spatial term on a level plane in 3-D space from an egocentric view.

\section{EXPERIMENT 3}

Experiment 2 showed that prototypical points of closed-area projective spatial terms such as ushiro could be computed using viewpoint and reference object positions on a level plane in 3-D space. However, the results of Experiment 2 may only have applied to ushiro. Therefore, we conducted Experiment 3 to ensure that our experimental assumptions could be applied to other spatial terms.

Experiment 3 replicated Experiment 2 using the Japanese spatial term mae. Mae has the opposite spatial meaning of ushiro and may be loosely translated as in front of in English.

\footnotetext{
${ }^{5}$ It is arguable what generally defines the essential difference between ushiro and saki. For example, the essential differences are defined by the frame of reference as shown in footnote \#3. This issue is important, but beyond the scope of this study.
} 
Table 4. Mean $(S D)$ ratios for each condition of mae

\begin{tabular}{cccc}
\hline \multicolumn{3}{c}{ Located object adjustment } & \\
\cline { 1 - 3 } Near & Middle & Far & \\
\hline $2.03(0.38)$ & $2.15(0.30)$ & $2.21(0.68)$ & $2.38(0.83)$ \\
\hline
\end{tabular}

Note. These values represent mean ratios of angles from the located object center to the view positions and angles from the reference object center to the view positions (located/reference).

\begin{abstract}
METHOD
Participants. Fifteen Japanese graduate or undergraduate students at Kyoto University participated in Experiment 3. All were native Japanese speakers who had not participated in Experiment 1 or 2.

Materials. The materials were the same as in Experiment 2, but the instructive sentence was changed to "Akai rippoutai-wa midori-no kyuu-no mae-ni aru" ("The red cube is in front of the green sphere").

Procedure. The procedure was the same as Experiment 2.
\end{abstract}

\title{
RESULTS
}

All raw data were processed as in Experiment 2. The ratios between the angles to the reference and located objects were computed for each condition (Table 4).

These ratios were subjected to one-way repeated measures ANOVAs on the viewing distance. In this procedure, the located object adjustment and distance adjustment conditions were grouped together; the viewing distance adjustment condition data were treated as a fourth dataset (in addition to near, middle, and far) of the located object adjustment condition. We found no significant main effect of viewing distance, and the ratios for mae was stable, $F(3,42)=1.036, p=.3864, M S E=0.308$.

\section{DISCUSSION}

The one-way repeated measure ANOVA on the ratios between angles from the viewpoint positions to the reference and located object positions indicated that the prototypical point of mae was relatively fixed to the reference object and likely to be defined by positions of the viewpoint and reference objects. As shown in Table 4, mean ratios for each viewing distance condition for mae were 2.03, 2.15, 2.21, and 2.38, respectively. The mean ratio of the four ratios was 2.19 in the 3-D CG space. As in Experiment 2, the following equation could be used to determine the prototypical point of mae in relation to the reference object.

$$
d_{n}=h \cot \left(\theta_{0} / 2.19\right)
$$

In the above equation, $h$ and $\theta_{0}$ are the parameters shown in Fig. 10 and $d_{n}$ is a predicted position of a prototypical point.

Experiment 3 revealed the same relationship between viewpoint and reference object positions as those found for ushiro in Experiment 2. This result supports the suggestion that prototypical points of closed-area projective spatial terms on a level plane in 3-D space can be successfully computed using the relationship between viewpoint and reference object positions in the given 3-D scene. 


\section{CONCLUSION}

We live in and use spatial terms in 3-D space; however, although various studies have examined means of mapping and computing the spatial characteristics of spatial terms (Carlson-Radvansky \& Logan, 1997; Crawford et al., 2000; Kojima, 2003; Logan \& Sadler, 1996; Munnich et al., 2001; Regier \& Carlson, 2000), few have focused on spatial terms on a level plane in 3-D space. Past studies have used 2-D upright displays and spatial terms that can be projected on a 2-D upright plane such as above, below, left and right, but have not considered depth-dependent terms such as behind, ahead, in front of and in back of, which are key spatial references in the real 3-D world. In 3-D space, a change in perspective changes in the observed scene. For instance, an apparent change in an object array is greater if the objects are arranged on a 3-D plane than on a 2-D upright plane.

This study examined whether, and how, we can compute a depth-dependent projective spatial term from an egocentric view. From previous studies on length or distance perception in 3-D space (Loomis et al., 1992; Norman \& Todd, 1998; Norman et al., 1996; Ooi et al., 2001; Philbeck \& Loomis, 1997; Wagner, 1985), we predicted that the relationship between viewpoint and object positions would be an important factor in the computation.

We chose ushiro (a closed-area projective spatial term) and saki (an open-area projective spatial term) as representative terms in Experiment 1 and 2. Experiment 1 showed an empirical difference between ushiro and saki (Fig. 5). For ushiro, the prototypical point was located on an axis and was closer to the reference object than the point for saki. In contrast, the prototypical point for saki was on an axis but could be farther from the reference object. From the results of Experiment 1, we defined two types of depth-dependent projective spatial terms: an open-area projective spatial term and a closed-area projective spatial term. Experiment 2 showed that it was possible to compute prototypical points of closed-area projective spatial terms by viewpoint and reference object positions.

Experiment 2 and 3 revealed that prototypical points of closed-area projective spatial terms such as ushiro and mae could be computed using viewpoint and reference object positions. The equations below generally compute an ideal point for a closed-area projective spatial term:

$$
\begin{aligned}
& \frac{\tan ^{-1}\left(h / d_{0}\right)}{\tan ^{-1}\left(h / d_{1}\right)}=\frac{\theta_{0}}{\theta_{1}}=r \\
& d_{n}=h \cot \left(\theta_{0} / r\right)
\end{aligned}
$$

where, $h, d_{0}, d_{1}, \theta_{0}$, and $\theta_{1}$ are the parameters shown in Fig. $10, d_{n}$ is a predicted position of a prototypical point determined by the equation, and $r$ is the ratio of a spatial term and constant in a specific spatial situation.

In each 3-D space, once parameter values were computed for a closed-area projective spatial term, the system could be used to choose an object indexed by that term. Because 
distance perception in 3-D CG space is distorted systematically, but is individually stable (Armbrüster et al., 2005), this model can be applied in the real world as well as to other closed-area projective spatial terms in various languages. However, there will be many problems to solve. Although this model treats objects as points, perceptual factors of objects, for example, size, shape, relative relationships between sizes or shapes, and so on, will have effects. In addition, factors of observers, for example, directions of their heads or eyes, somatic sensation and so on, will also be important. These factors may demand new parameters on the model. Further research should be conducted to improve the model and gather more data for specific or general applications.

We assumed that for saki, an open-area projective spatial term, a prototypical location or point could be computed using a vanishing point of an observed scene and a reference object position. However, because we did not examine how humans recognize a vanishing point from an observed scene, we could not show this relationship from the present study. Further examination of this issue will be a topic in future research.

The real world provides rich perceptual information. Though we cannot always use all the information available in apprehending spatial terms or expressions, our spatial indexing usually allows effective communication among individuals, assuming that spatial term apprehension is generally shared within a specific language or culture. Therefore, the prototypical positions of some spatial terms can be computed on a level plane in 3-D space, as shown in this study.

According to Logan and Sadler (1996), the linguistic apprehension of spatial relationship generally consists of four processes: spatial indexing, reference frame adjustment, spatial template alignment and computing goodness of fit for the spatial template. Spatial indexing binds objects in a statement to objects in the perceptual representation. Reference frame adjustment adjusts a reference frame in perceptual representation by setting four parameters: origin, orientation, direction, and scale. Spatial template alignment imposes a spatial template on a reference object and aligns it within a reference frame. Computing goodness of fit computes the overall acceptable pattern of a spatial template and determines good, acceptable and bad regions. Computing goodness of fit may parallel the whole visual (or imagined) field. However, complicated changes in egocentrically observed scenes in 3-D space, and our results, suggest that a prototypical point on a spatial template is first detected from an observed scene, and then an overall goodness-of-fit pattern spreads out from the reference object and ideal point.

Experiment 2 and 3 showed that the ratios between the ascending angles from a viewpoint to the reference and located objects were constant for ushiro and mae. Thus, perceptual representation in 3-D space cannot be apprehended through Euclidean-based means. The origins of perceptual and linguistic spatial representations have created much controversy, and various schools of thought have arisen around this, including theories based on the idea that perceptual spatial representation constitutes the basis for linguistic spatial representation (Landau \& Jackendoff, 1993; Mandler, 1992; Talmy, 1983), that linguistic spatial representation forms the basis for perceptual spatial representation (Levinson, 1996; Lucy, 1992; Whorf, 1956), or that each type of representation forms the basis for the other (Yamanashi, 2000). In any case, the relationship between spatial terms 
and spatial representation remains one of the most important issues in psychology and linguistics. Some linguists have assumed that such relationships can be depicted on 2-D planes, as in overhead or lateral views (e.g., Kreitzer, 1997; Lakoff, 1987; Talmy, 1983; Tyler \& Evans, 2003). However, the present research was based on the assumption that ideal spatial images for depth-dependent terms such as ushiro and mae, (behind or in back of and in front of in English) could not be depicted on 2-D planes.

Although we used 3-D CG space and limited many factors, which may not be possible in real-world situations, this study should be helpful to future studies on the relationship between language and virtual/real space. Even though the 3-D CG space of this study was relatively unfamiliar to participants, they firmly abstracted the relationship between the ideal points of ushiro and mae. This interesting result points to the usefulness of this method to applied spatial cognition and computation research. Methods employed in this study could also be used to improve the interface of various systems that contain 3D CG displays and language-based instruction, such as the 3-D avatar chat systems and video games, which have become popular virtual Internet destinations. Understanding the relationships between spatial terms and spatial representation in a 3-D virtual space is thus of growing importance for both basic and applied studies.

\section{REFERENCES}

Armbrüster, C., Heber, I. A., Valvoda, J. T., Kuhlen, T., \& Spijkers, W. 2005. Distance estimation in a VR application: Interindividual differences and intraindividual stabilities from a psychological point of view. Proceedings of the 11th International conference on Human-Computer Interaction, CD-ROM.

Carlson, L. A., \& Logan, G. D. 2001. Using spatial terms to select an object. Memory \& Cognition, 29, 883892.

Carlson-Radvansky, L. A., Covey, E. S., \& Lattanzi, K. M. 1999. "What" effects on "where": Functional influences on spatial relations. Psychological Science, 10, 516-521.

Carlson-Radvansky, L. A., \& Logan, G. D. 1997. The influence of reference frame selection on spatial template construction. Journal of Memory and Language, 37, 411-437.

Crawford, L. E., Regier, T., \& Huttenlocher, J. 2000. Linguistic and non-linguistic spatial categorization. Cognition, 75, 209-235.

Creem-Regehr, S. H., Willemsen, P., Gooch, A. A., \& Thompson, W. B. 2005. The influence of restricted viewing conditions on egocentric distance perception: Implication for real and virtual indoor environments. Perception, 34, 191-204.

Franklin, N., Henkel, L. A., \& Zengas, T. 1995. Parsing surrounding space into regions. Memory \& Cognition, 23, 379-407.

Hayward, W. G., \& Tarr, M. J. 1995. Spatial language and spatial representation. Cognition, 55, 39-84.

Herskovits, A. 1986. Language and spatial cognition: An interdisciplinary study of the prepositions of English. Cambridge: Cambridge University Press.

Imai, M., Nakanishi, T., Miyashita, H., Kidachi, Y., \& Ishizaki, S. 1999. The meanings of front/back/left/ right. Cognitive Studies, 6, 207-225.

Kojima, T. 2003. A method for testing spatial structures of spatial terms [Abstract]. Proceedings of the 25th annual Conference of the Cognitive Science Society, 1366.

Kojima, T., \& Kusumi, T. 2002. The structure of linguistic spatial representation: A test for psychometric structure using Japanese spatial terms [Abstract]. Proceedings of the 24th annual Conference of the Cognitive Science Society, 1013.

Kreizer, A. 1997. Multiple levels of schematization: A study in the conceptualization of space. Cognitive Linguistics, 8, 291-325. 
Lakoff, G. 1987. Women, fire and dangerous things: What categories reveal about the mind. Chicago: Chicago University Press.

Landau, B., \& Jackendoff, R. 1993. "What" and "where" in spatial language and spatial cognition. Behavioral and Brain Sciences, 16, 217-265.

Levinson, S. C. 1996. Frames of Reference and Molyneux's question: cross linguistic evidence. In P. Bloom, M.A. Peterson, L. Nadel, \& M. F. Garrett, Language and space (pp. 109-170). Cambridge, MA: MIT Press.

Logan, G.D., \& Compton, B. 1996. Distance and distraction effects in the apprehension of spatial relations. Journal of Experimental Psychology: Human Perception \& Performance, 22, 159-172.

Logan, G. D., \& Sadler, D. D. 1996. A computational analysis of the apprehension of spatial relations. In P. Bloom, M.A. Peterson, L. Nadel, \& M. Garrett (Eds.), Language and space (pp. 493-529). Cambridge, MA: MIT Press.

Loomis, J. M. Da Silva, J. A., Fujita, N., \& Fukushima, S. S. 1992. Visual space perception and visually directed action. Journal of Experimental Psychology: Human Perception and Performance, 18, 906921.

Lucy, J. 1992. Language diversity and thought: a reformulation of the linguistic relativity hypothesis. Cambridge, MA: MIT Press.

Mandler, J. M. 1992. How to build a baby: II .Conceptual Primitives. Psychological Review, 99, 587-604.

Miller, G. A., \& John-son Laird, P. N. 1976. Language and perception. Cambridge, MA: Belknap Press.

Munnich, E., Landau, B., \& Dosher, B. A. 2001. Spatial language and spatial representation: a crosslinguistic comparison. Cognition, 81, 171-207.

Norman, J. F., Lappin, J. S., \& Norman, H. F. 2000. The perception length on curved and flat surfaces. Perception \& Psychophysics, 62, 1133-1145.

Norman, J. F., Norman, H. F., Lee, Y. M., \& Stockton, D. 2004. The visual perception of length along intrinsically curved surfaces. Perception \& Psychophysics, 66, 77-88.

Norman, J. F., Todd, J. T. 1998. Stereoscopic discrimination of interval and ordinal depth relations on smooth surfaces and in empty space. Perception, 27, 257-272.

Norman, J. F., Todd, J. T., Perotti, V. J., \& Tittle, J. S. 1996. The visual perception of three-dimensional length. Journal of Experimental Psychology: Human Perception and Performance, 22, 173-186.

Ooi, T. L., Wu, B., \& He, Z. J. 2001. Distance determined by the angular declination below the horizon. Nature, 414, 197-200.

Philbeck, J. W., \& Loomis, J. M. 1997. Comparison of two indicators of perceived egocentric distance under full-cue and reduced-cue conditions. Journal of Experimental Psychology: Human Perception and Performance, 23, 72-85.

Regier, T. 1996. The human semantic potential: Spatial language and constrained connectionism. Cambridge, MA: MIT Press.

Regier, T., \& Carlson, L. A. 2001. Grounding spatial language in perception: An empirical and computational investigation. Journal of Experimental Psychology: General, 130, 273-298.

Talmy, L. 1983. How language structures space. In H. L. Pick., \& L. P. Acredolo (Eds.). Spatial orientation: Theory, research, and application (pp. 225-282). New York: Plenum.

Tokunaga, T., Koyama, T., \& Saito, S. 2005. Meaning of Japanese spatial nouns. Proceedings of the 2nd ACL-SIGSEM workshop on the Linguistic Dimensions of Prepositions and their Use in Computational Linguistics Formalisms and Applications, 93-100.

Tyler, A., \& Evans, V. 2003. The semantics of English prepositions: Spatial scenes, embodied meaning and cognition. Cambridge: Cambridge University Press.

Wagner, M. 1985. The metric of visual space. Perception \& Psychophysics, 38, 483-495.

Whorf, B. 1956. Language, thought and reality. New York: Technology Press of MIT and Wiley.

Yamanashi, M. 2000. Principle of cognitive linguistics. Tokyo: Kuroshio Press. 